\title{
Prediction of internal carotid artery aneurysm recurrence by pressure difference at the coil mass surface
}

\author{
Takehiro Uno $^{1}$ (D) Kouichi Misaki $^{1}$ (D) Iku Nambu $^{1}$ (D) $\cdot$ Akifumi Yoshikawa ${ }^{1} \cdot$ Tomoya Kamide $^{1}$ (D) \\ Naoyuki Uchiyama ${ }^{1}$ (1) $\cdot$ Mitsutoshi Nakada ${ }^{1}$ (i)
}

Received: 16 June 2020 / Accepted: 8 September 2020 / Published online: 14 September 2020

(C) The Author(s) 2020

\begin{abstract}
Purpose A previous study on computational fluid dynamics reported that a high pressure difference (PD) at the surface of a coil mass is a strong predictor of aneurysm recurrence after coil embolization. PD was calculated using a virtual post-coiling model (VM), created by manually cutting the aneurysm by the flat plane from an anatomic model created with pre-coil embolization data; however, its credibility has not been fully evaluated. This study aims to clarify whether PD values calculated using the postcoiling model, which reflects the actual coil plane, are a strong predictor of aneurysm recurrence.

Methods Fifty internal carotid artery aneurysms treated with endovascular coil embolization were analyzed ( 7 recanalized, 43 stable). We created and subjected two post-coiling models, namely, VM and the real post-coiling model (RM), constructed from the post-coil embolization data. The relationship between PD and aneurysm recurrence was examined using these models. PD and its constituent three parameters were compared between VM and RM.

Results PD values calculated using RM showed significantly higher aneurysm recurrence in recurrence group than stable group $(p<0.001)$, and multivariate analysis showed that PD in RM $(p=0.02$; odds ratio, 36.24) was significantly associated with aneurysm recurrence. The receiver operating characteristic analysis revealed that PD values accurately predicted aneurysm recurrence (area under the curve, 0.977; cutoff value, 3.08; sensitivity, 100\%; specificity, 97.7\%). All four parameters showed a significant correlation with VM and RM $(p<0.001)$.

Conclusion Use of PD to predict recurrence after coil embolization can be clinically relevant.
\end{abstract}

Keywords Pressure difference $\cdot$ Virtual post-coiling model $\cdot$ Intracranial aneurysm $\cdot$ Computational fluid dynamics $\cdot$ Recurrence after coil embolization

\begin{tabular}{|c|c|}
\hline \multicolumn{2}{|c|}{ Abbreviations } \\
\hline 3D-RA & $\begin{array}{l}\text { Three-dimensional rotational } \\
\text { angiography }\end{array}$ \\
\hline AUC & Area under the curve \\
\hline CE-MRA & $\begin{array}{l}\text { Contrast-enhanced magnetic } \\
\text { resonance angiography }\end{array}$ \\
\hline \multirow{2}{*}{$\begin{array}{l}\text { CFD } \\
\text { DICOM }\end{array}$} & Computational fluid dynamics \\
\hline & $\begin{array}{l}\text { Digital imaging for communication } \\
\text { in medicine }\end{array}$ \\
\hline DSA & Digital subtraction angiography \\
\hline \multicolumn{2}{|c|}{$\begin{array}{l}\triangle \text { Kouichi Misaki } \\
\text { misaki@med.kanazawa-u.ac.jp }\end{array}$} \\
\hline \multicolumn{2}{|c|}{$\begin{array}{l}\text { Department of Neurosurgery, Graduate School of Medical Science, } \\
\text { Kanazawa University, 13-1 Takara-machi, } \\
\text { Kanazawa, Ishikawa 920-8641, Japan }\end{array}$} \\
\hline
\end{tabular}

$\begin{array}{ll}\text { ICA } & \text { Internal carotid artery } \\ \text { PcomA } & \text { Posterior communicating artery } \\ \text { PD } & \text { Pressure difference } \\ \text { RM } & \text { Real post-coiling model } \\ \text { ROC } & \text { Receiver operating characteristic } \\ \text { TOF-MRA } & \text { Time-of-flight magnetic } \\ & \text { resonance angiography } \\ \text { VER } & \text { Volume embolization ratio } \\ \text { VM } & \text { Virtual post-coiling model }\end{array}$

\section{Introduction}

Endovascular coil embolization is a safe and reliable technique for intracranial aneurysm treatment $[1,2]$. However, aneurysms are more likely to recur after coil embolization than after surgical clipping [3-6]. It is crucial to investigate the recurrence rate after 
coil embolization and actively assess it in the field of computational fluid dynamics (CFD) [7, 8]. Recently, Nambu et al. [9] thoroughly investigated the risk factors for aneurysm recurrence after coil embolization by conducting CFD analysis using the virtual post-coiling model (VM). VM was constructed from the pre-coil embolization data and by manually cutting the aneurysm by the flat plane, thus dividing the aneurysm dome and the origin of the branching vessel or parent artery. They concluded that a high pressure difference (PD) was the strongest predictor for aneurysm recurrence when compared with other morphological and hemodynamic parameters. This result is beneficial because it enables predicting aneurysm recurrence before treatment. However, in the VM, part of the aneurysm was artificially deleted, and the surface of the coil mass, defined as the coil plane, was a virtual plane. Furthermore, the virtual coil plane is morphologically different from the actual coil plane in that it is rough; however, the reliability of VM is unknown. It is necessary to examine whether the same tendency can be obtained using the model reflecting the actual coil plane. We constructed a real postcoiling model (RM), reflecting the actual coil plane, from the post-coil embolization data. In this study, we investigated the role of PD in predicting aneurysm recurrence after coil embolization using VM and RM, and we compared the results of both models.

\section{Methods}

\section{Source of the patient data}

Among patients who underwent endovascular coil embolization for internal carotid artery (ICA) aneurysm treatment at our institution, we selected those who underwent timeof-flight magnetic resonance angiography (TOF-MRA) imaging at follow-up. In particular, we focused on patients who have been followed up for over 1 year and whose radiological data quality has been maintained sufficiently for CFD analysis. All patients had a volume embolization ratio (VER), the ratio of the volume of the aneurysm to the volume of the coil, of at least $20 \%$. Finally, 48 patients with 50 ICA aneurysms (32 in the posterior communicating artery, 11 in the paraclinoid ICA, 4 in the ophthalmic artery, and 3 in the anterior choroidal artery) were included in the study. Of the 50 cases, 15 were ruptured, and 35 were unruptured aneurysms. In this study, recanalized aneurysms were defined as cases with an increase in the Raymond-Roy grade or cases within the grading but required retreatment. We then defined stable aneurysm as cases with no change or enlargement within a grade with no retreatment. The institutional review board approved this study, and prior informed consent was obtained from the patients.

\section{Aneurysm modeling}

For each aneurysm, both VM and RM were created (Fig. 1). We used the digital imaging for communication in medicine (DICOM) data of three-dimensional rotational angiography (3D-RA) for VM analysis and the TOF-MRA data for RM analysis. TOF-MRA was performed at $1.5 \mathrm{~T}$ (SIGNA HDxt; GE Medical Systems, Milwaukee, WI) or 3 T (GE Healthcare, Milwaukee) field strengths. TOF-MRA imaging parameters were as follows: for $1.5 \mathrm{~T}, 25 \mathrm{~ms}$ repetition time (TR), $3.2 \mathrm{~ms}$ echo time (TE), 1 number of signal averages (NSA), $1.2 \mathrm{~mm}$ section thickness, $200 \mathrm{~mm}$ field of view (FOV), and $320 \times 192$ acquisition matrix, and for $3 \mathrm{~T}, 24 \mathrm{~ms}$ TR, $3.4 \mathrm{~ms}$ TE, $0.85 \mathrm{NSA}, 1 \mathrm{~mm}$ section thickness, $200 \mathrm{~mm}$ FOV, and $384 \times 224$ acquisition matrix. Vascular geometry was extracted using manual cropping and image thresholding. When identifying the boundaries of the geometry of blood vessels from the TOF-MRA and 3D-RA voxel data, the 2-D digital subtraction angiography (DSA) geometry was checked to ensure that the geometry was as close as possible to the reality. Aneurysm and main blood vessels were converted into standard triangulated surfaces using Amira (version 5.6, Maxnet Co. Ltd., Tokyo, Japan). These 3-D images were imported into ICEM CFD software (version 16.2, ANSYS Inc., Canonsburg, Pennsylvania, USA) to construct the aneurysm and blood vessel structures [10]. When creating a blood vessel model, care was taken to maintain the proper length of the mother blood vessel to maintain accurate quality of the numerical simulation.

VM was created using DICOM image data from 3D-RA, which was imaged before coiling. The coil plane in VM was set to a plane where the aneurysm neck and branch blood vessels or parent artery can be separated under the assumption that complete obliteration was achieved. We artificially deleted part of the aneurysm, and the surface had a virtual coil plane, thus creating a VM (Fig. 1a-c). After coiling (Fig. 1d), the RM was created using the DICOM image data from the TOF-MRA images after treatment (Fig. 1e). We used the data that were obtained within a week from coiling to MRA imaging. Unlike VM, where the aneurysm was artificially deleted to create a flat virtual coil plane, RM was created using the actual postoperative DICOM data. The coil plane of the RM was an uneven surface reflecting the actual effect of the coil mass (Fig. 1f). The inlet plane was defined as the section plane of the ICA located $1 \mathrm{~mm}$ proximal to the aneurysm, as previously described $[10,11]$.

\section{Numerical simulations}

We performed CFD simulations as previously described [9, $12,13]$. ANSYS ICEM CFD software was used to create fluid domains for the two vascular models for each patient in order to create meshes with compromised tetrahedrons and seven 

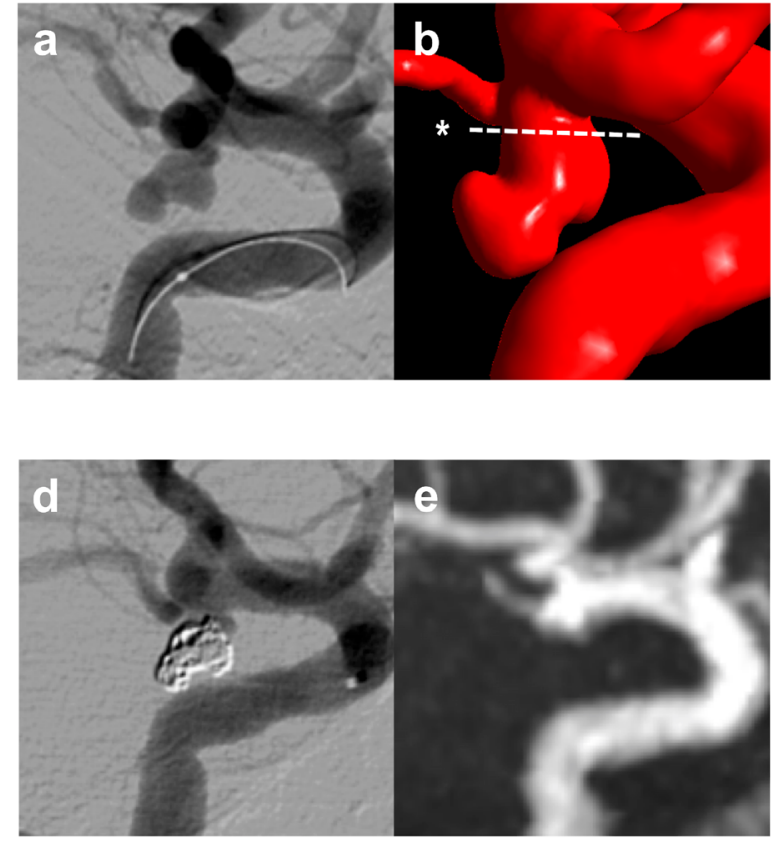

Fig. 1 The virtual post-coiling model (VM) constructed using the digital imaging for communication in medicine (DICOM) data of pre-coil embolization three-dimensional rotational angiography (a). First, a precoiling model is constructed by artificially deleting part of the aneurysm at the coil plane (b) $\left(^{*}\right)$. The coil plane of VM is flat (c). The twodimensional digital subtraction angiography of the final image after coil

prism element layers near the wall surface in the boundary. A 75-mm passage was added to the proximal plane of the vessel structure to make the inlet length sufficiently long for simulation [8]. Blood density and dynamic viscosity were defined as $1100 \mathrm{~kg} / \mathrm{m} 3$ and $0.0036 \mathrm{~Pa}$ s, respectively, and were modeled as Newtonian fluids. The condition of the vessel wall was assumed to be a hard slip-free boundary. To solve the pulsatile-flow governing Navier-Stokes equations, we used ANSYS CFX (version 16.2, ANSYS Inc.) [14]. In this study, we used average mass flow rates in the ICA measured from healthy adults, as described by Ford et al., and then a 1.8-s transient analysis was performed [15]. The pressure was set to zero at the outlet, and the same boundary conditions were applied to all models. The calculation interval time was set to $0.005 \mathrm{~s}$, and two cardiac cycles were simulated. The result of the second cycle was used in this study.

\section{Analysis of hemodynamic parameters in the virtual and real post-coiling models}

We examined 4 hemodynamic parameters in both VM and RM. The maximum pressure (Pmax) at the coil plane, average pressure (Pave) at the inlet plane, average velocity (Vin) at the inlet plane, and PD were measured [9]. Pmax, Pave, and Vin are components of PD. In this study, we examined these 4 hemodynamic parameters to investigate the relationship

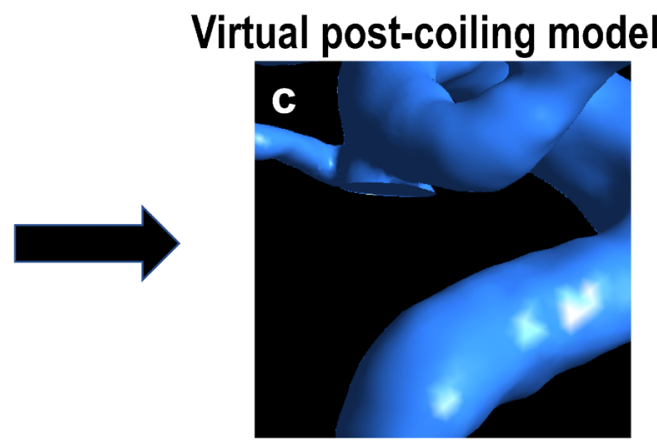

\section{Real post-coiling model}

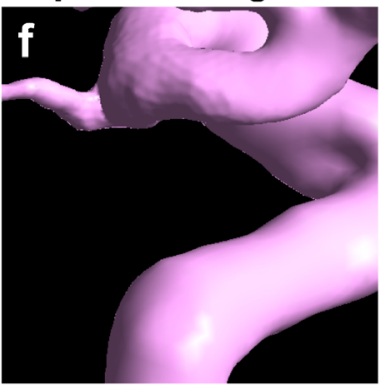

embolization is shown (d). The real post-coiling model (RM) constructed the DICOM data of post-coil embolization time-of-flight magnetic resonance angiography (e). It is created using the data on the actual coil plane obtained from the data after the actual coil embolization. The coil plane of $\mathrm{RM}$ is rough and uneven (f)

between PD and aneurysm recurrence using VM and RM. Pmax was calculated as the highest value of pressure in the virtual and real coil planes. Pave was calculated as the mean value of the pressure at the inlet plane. Vin was calculated as the average flow velocity at the inlet plane in meters per second. The PD in this study was calculated using the following equation:

$$
\text { Pressure difference }=\frac{\text { Pmax }-\mathrm{P} \text { ave }}{\frac{1}{2} \rho \operatorname{Vin}^{2}}
$$

In this equation, $\rho$ is $1100 \mathrm{~kg} / \mathrm{m} 3$. The pressure difference was defined as the degree of pressure elevation at the postcoiling plane from the inlet plane calculated by subtracting Pave from Pmax. This value is normalized by dividing the dynamic pressure at the inlet plane. These values were analyzed at the peak systole.

\section{Statistical analysis}

Continuous data and the number of observations were reported as mean \pm standard deviations for continuous variables (frequency, \%). Mann-Whitney $U$ test or Fisher's exact test was used to analyze parameters, as appropriate. Hemodynamic parameters were predicted by constructing receiver operating characteristic (ROC) curves and calculating 
the area under the curve (AUC). Univariate logistic regression model was estimated in order to identify predictive factors of recurrence after coil embolization. Variables statistically significant at univariate analysis were then included in the multivariate logistic regression model to identify independent predictive factors of recurrence after coil embolization. However, Of the values that showed statistically significant different in univariate analysis, $\mathrm{PD}$ in VM is a factor similar to PD in RM, and the value of PD in VM, which was considered to be less correlated with recurrence than PD in RM from the ROC curve, was excluded from consideration. Spearman's rank correlation test was used to determine numeric relationships between the values calculated using VM and those calculated using RM. Statistical significance was indicated when $p<0.05$. Statistical analysis was performed using SPSS (IBM SPSS Statistics 24, Chicago, IL, USA).

\section{Results}

Seven of the 50 aneurysms (14.0\%) were classified into the recanalized aneurysm group. All of them were located at the posterior communicating artery (PcomA). Comparisons of patients' and morphological and hemodynamic characteristics between the recanalized group and the stable group are shown in Table 1. For morphological features, maximum size, and percentage of ruptured aneurysms were significantly higher in the recanalized group than in the stable group. There was no significant difference in the association of PcomA with fetal type and recurrence. Regarding hemodynamic parameters, no significant differences were found in terms of the three components of PD; however, PD was significantly higher in the recanalized aneurysm group than in the stable aneurysm group. VM and RM showed a similar tendency. Only PD in RM (odds ratio: $36.24,95 \%$ confidence interval $1.47-893.69 ; p=0.02$ ) showed a statistically significant difference when multivariate logistic regression analysis was performed on values including maximum size, PcomA aneurysm, ruptured aneurysm and PD in RM. When we focused on the value calculated using VM and RM (Fig. 2), PD in VM was $3.60 \pm 0.78$ in the recurrence group, significantly higher than that of $2.15 \pm 0.64$ in the stable group $(p<0.001)$, and PD in RM was $3.40 \pm 0.24$ in the recurrence group, significantly higher than that of $1.99 \pm 0.75$ in the stable group $(p<0.001$, Fig. 2a). The ROC analysis for PD in VM and RM revealed an AUC of 0.967 , a sensitivity of $100 \%$, and a specificity of $90.7 \%$ when the cutoff value was 2.83 in VM and AUC of 0.977 , a sensitivity of $100 \%$, and a specificity of $97.7 \%$ when the cutoff value was 3.08 in RM, respectively (Fig. 2b). Similar to the VM results, cases with a high PD also recurred after coil embolization in the RM results. PD,
Pmax, Pave, and Vin showed significant correlation with $\mathrm{VM}$ and RM $(p<0.001$, Fig. 3$)$. The correlation coefficient was 0.698 for PD, 0.559 for Pmax, 0.561 for Pave, and 0.537 for Vin.

\section{Illustrative cases}

\section{Recanalized case}

A ruptured PcomA aneurysm was found in a 66-year-old woman (Fig. 4). The largest diameter of this aneurysm was $10.8 \mathrm{~mm}$ (Fig. 4a). This aneurysm was treated by coil embolization with $20.6 \%$ of the final VER (Fig. 4b) and recanalized after 6 months (Fig. 4c). The coil planes of VM and RM are demonstrated in Fig. $4 \mathrm{~d}$ and g, respectively. The contour lines of the pressure difference are depicted on the coil plane of the VM (Fig. 4e) and RM (Fig. 4h). A high PD area was present near the branch vessels in both models. Streamlines of VM (Fig. 4f) and RM (Fig. 4i) are shown in the figures. Blood flow collided with the coil planes and diverged to the branch vessels and parent arteries in both models. The PD values in this case were 3.05 and 3.43 in VM and RM, respectively.

\section{Stable case}

An unruptured paraclinoid aneurysm was diagnosed in a 44year-old man (Fig. 5). The largest diameter of this aneurysm was $9.4 \mathrm{~mm}$ (Fig. 5a). This aneurysm was treated using coil embolization with $23.3 \%$ of the final VER (Fig. 5b) and did not recanalize at 12 months after treatment (Fig. $5 \mathrm{c}$ ). The coil planes of VM and RM are shown in Fig. 5d and g. The contour lines of the pressure difference are depicted on the coil plane of VM (Fig. 5e) and RM (Fig. 5h). A high PD area was present on the proximal side of the coil plane. Streamlines of VM (Fig. 5f) and RM (Fig. 5i) are shown in the figures. Blood flow was observed along the coil plane in both models. The PD values of this case were 2.30 and 2.80 in VM and RM, respectively.

\section{Discussion}

We investigated for recurrent factors after coil embolization with RM constructed from the TOF-MRA data after coil embolization. Nambu et al. reported a significantly higher PD value in recurrent cases using VM and an AUC of 0.967, a sensitivity of $100 \%$, and a specificity of $90.7 \%$, when the cutoff value was 2.83 in ROC analysis. The study concluded that among the seven items examined, PD values calculated using VM showed the highest correlation with recurrence and were the strongest predictor of recurrence after coil embolization [9]. PD values calculated using RM were significantly 
Table 1 Patient and morphological and hemodynamic characteristics

\begin{tabular}{|c|c|c|c|c|c|c|}
\hline & \multicolumn{3}{|l|}{ Univariate analysis } & \multicolumn{3}{|c|}{ Multivariate analysis } \\
\hline & Recanalized group $(n=7)$ & Stable group $(n=43)$ & $p$ value* & OR & $95 \% \mathrm{CI}$ & $p$ value \\
\hline Sex, $n(\%)$ & & & 0.14 & & Not evaluated & \\
\hline Male & $2(28.6)$ & $3(7.0)$ & & & Not evaluated & \\
\hline Female & $5(71.4)$ & $40(93.0)$ & & & Not evaluated & \\
\hline Age, years & $65 \pm 14$ & $58 \pm 13$ & 0.20 & & Not evaluated & \\
\hline Maximum size, mm & $11.5 \pm 2.6$ & $8.3 \pm 2.5$ & 0.01 & & & 0.20 \\
\hline PcomA aneurysm (\%) & $7(100)$ & $25(58.1)$ & 0.04 & & & 0.99 \\
\hline Ruptured aneurysm (\%) & $5(71.4)$ & $10(23.3)$ & 0.02 & & & 0.07 \\
\hline VER, \% & $22.5 \pm 3.50$ & $24.8 \pm 4.5$ & 0.16 & & Not evaluated & \\
\hline Pmax in $\mathrm{VM}, \mathrm{Pa}$ & $3815.09 \pm 3014.30$ & $4440.71 \pm 4597.14$ & 0.89 & & Not evaluated & \\
\hline Pave in $\mathrm{VM}, \mathrm{Pa}$ & $3679.95 \pm 3031.15$ & $4267.22 \pm 4554.09$ & 0.81 & & Not evaluated & \\
\hline Vin in $V M, m / s$ & $0.63 \pm 0.17$ & $0.66 \pm 0.20$ & 0.81 & & Not evaluated & \\
\hline $\mathrm{PD}$ in VM & $3.60 \pm 0.78$ & $2.15 \pm 0.64$ & $<0.001$ & & Not evaluated & \\
\hline Pmax in $\mathrm{RM}, \mathrm{Pa}$ & $6236.32 \pm 4801.38$ & $10,617.80 \pm 16,704.73$ & 0.83 & & Not evaluated & \\
\hline Pave in $\mathrm{RM}, \mathrm{Pa}$ & $5672.88 \pm 4789.61$ & $10,039.57 \pm 16,397.22$ & 0.74 & & Not evaluated & \\
\hline Vin in $R M, m / s$ & $0.54 \pm 0.10$ & $0.70 \pm 0.31$ & 0.41 & & Not evaluated & \\
\hline $\mathrm{PD}$ in $\mathrm{RM}$ & $3.40 \pm 0.24$ & $1.99 \pm 0.75$ & $<0.001$ & 36.24 & $1.47-893.69$ & 0.02 \\
\hline
\end{tabular}

PcomA, posterior communicating artery; VER, volume embolization ratio; Pmax, maximum pressure at coil plane; Pave, average pressure at inlet plane; Vin, mean velocity of at inlet; PD, pressure difference; VM, virtual post-coiling model; RM, real post-coiling model; OR, odds ratio; $95 \%$ CI, $95 \%$ confidence interval. Values are shown as mean $\pm \mathrm{SD}$ when appropriate

*The $t$ test and Mann-Whitney $U$ test were used; $p<0.05$ was considered statistically significant

higher in recurrence cases. Additionally, the calculated values using RM showed a significant correlation with VM with respect to $\mathrm{PD}$, and the three parameters were required for the PD calculation. Our results suggest that whether calculated

\section{a}

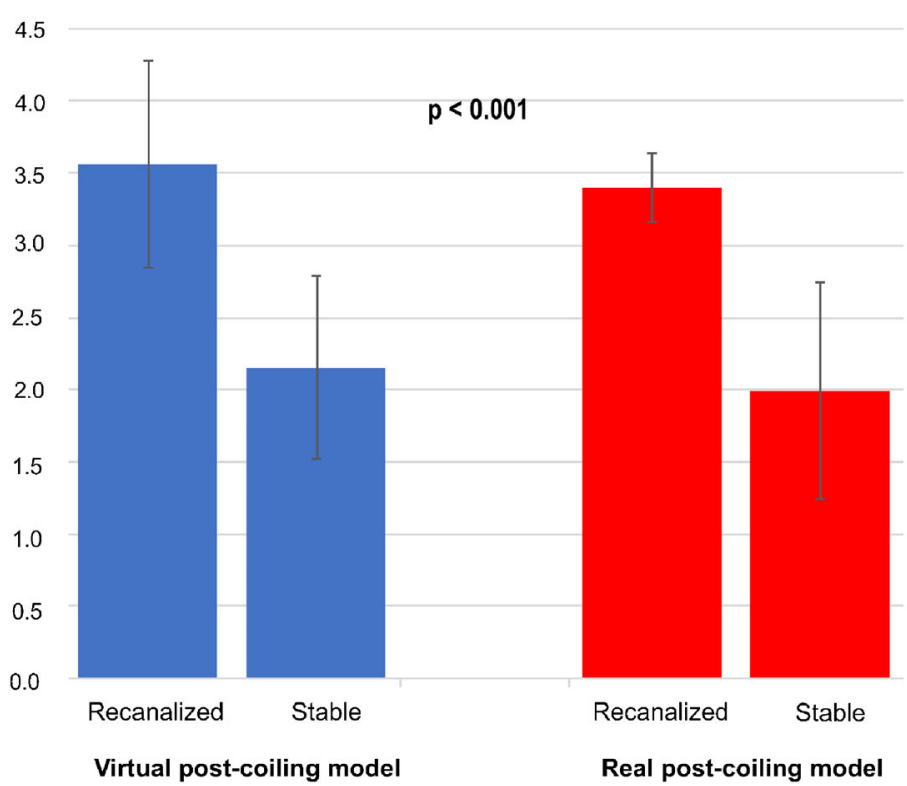

Fig. 2 The relationship between pressure difference (PD) and recanalized aneurysm in the virtual post-coiling model and real post-coiling model. a Comparison of PD values between the recanalized and stable groups. $\mathbf{b}$ using VM or RM, PD shows a strong association with recurrence and is a useful factor for predicting recurrence.

As for the characteristics of patients in this study, maximum aneurysm size, PcomA aneurysm, and ruptured

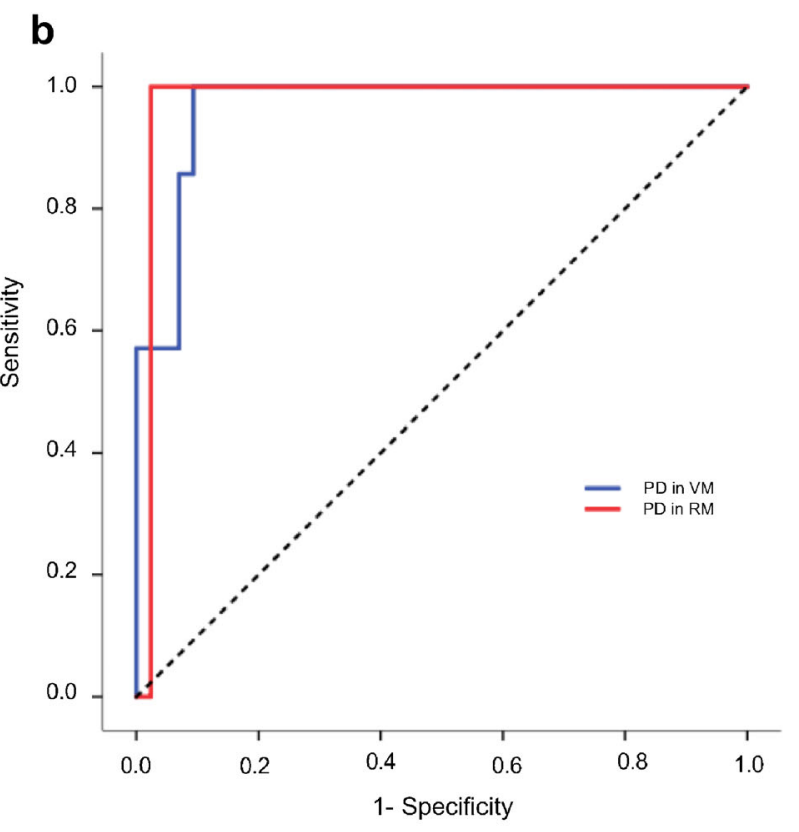

Receiver operating characteristic curve of PD for predicting recanalization after coil embolization in the coil plane. AUC, area under the curve 


\section{a Maximum pressure at coil plane}

은

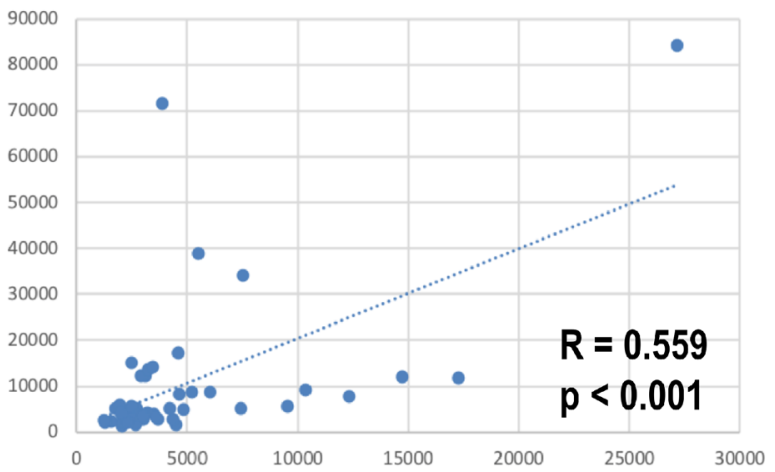

Virtual post-coiling model $[\mathrm{Pa}]$

\section{c Average velocity at inlet plane}
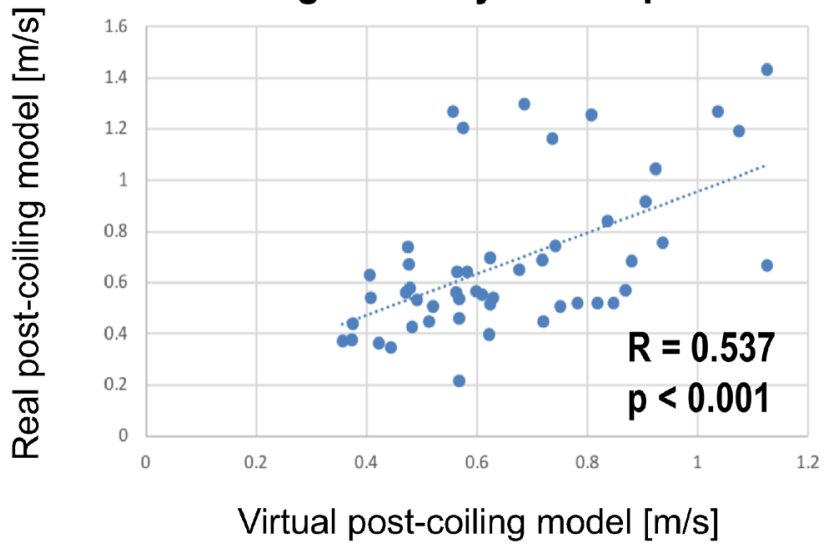

Fig. 3 Examples of correlation charts between pressure difference and the three components of pressure difference for virtual post-coiling model and real post-coiling model. a Maximum pressure at the coil plane. b

aneurysm were significantly higher in the recurrence group than in the stable group. Raymond et al. reported that variables that were significant predictors of recurrence included aneurysm size $\geq 10 \mathrm{~mm}$ and treatment during the acute phase of rupture [4]. Furthermore, Compi et al. found that coiled aneurysms in the PcomA location had a significantly increased risk of recurrence requiring re-embolization [16]. The characteristics of the patients in this study were similar to those reported previously.

Studies have used VM created by artificially deleting vascular structures from vascular models [9, 17-19]. In these reports, hemodynamic factors such as influence of bleb formation, aneurysm rupture, and recurrence factors after coil embolization were examined. Although VM are models in which structures are artificially deleted, it has not been clarified whether examination in the model reflects an actual in vivo phenomenon. There have been no reports on the verification of VM use for this purpose thus far, and its credibility has not been validated. We investigated the reliability of using VM by comparing the results of VM with those of RM. The

\section{b Average pressure at inlet plane}

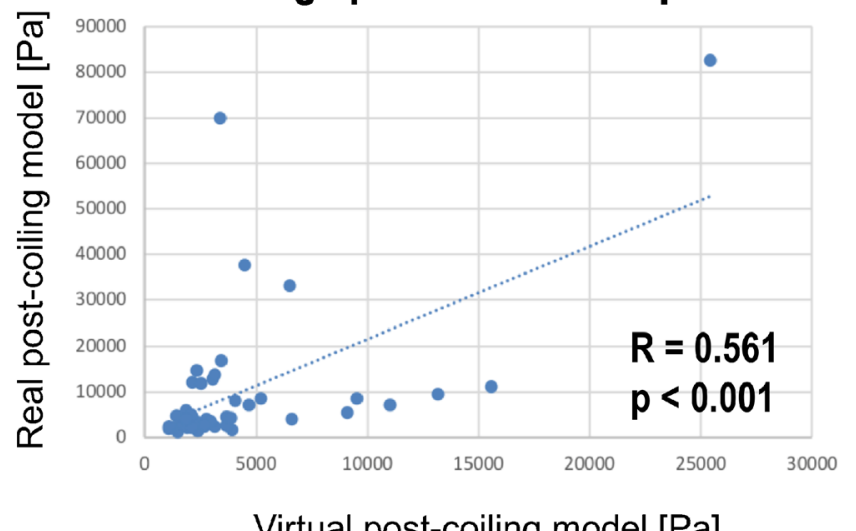

\section{d Pressure difference}

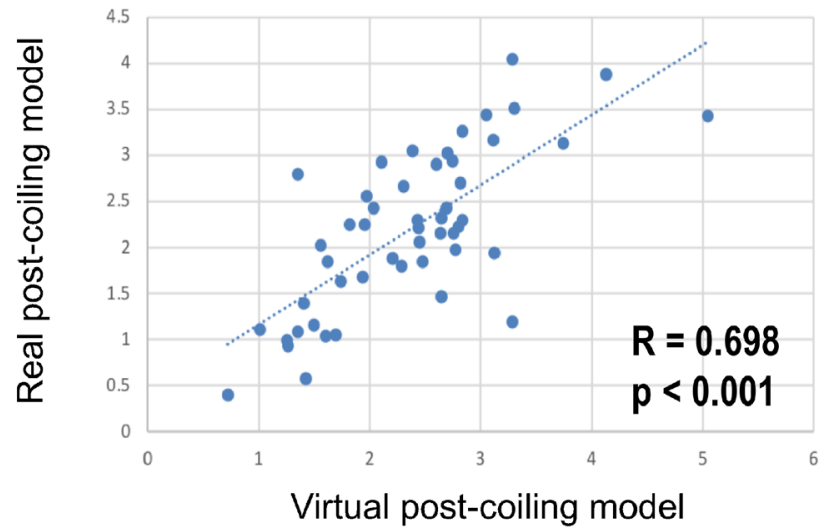

Average pressure at the inlet plane. c Average velocity at the inlet plane. d Pressure difference

results of this study indicate that VM and RM show significant correlation, and these two models are useful for the examination of hemodynamic factors.

Standardizing parameters is a universal index because it standardizes and examines individual differences between each case. A number of studies have examined standardized parameters in the past. PD is a non-dimensionalized value, obtained by dividing the pressure increase from the mother blood vessel to the coil plane by the dynamic pressure. PD can be defined as the degree of pressure increase from the mother blood vessel at the coil plane, which generalizes individual differences among patients $[9,20,21]$. As we adopted the inlet conditions uniformly, parameter values are preferable to be standardized values to account for patient-specific inflow conditions. We speculate that high PD on the coil mass surface may cause coil compaction, resulting in the recurrence of treated aneurysms [22].

PD can be calculated using VM before treatment and is useful for treatment planning. Patients with high PD calculated using VM may need to consider changes in treatment 
Fig. 4 Illustrative recurrence case of a 66-year-old woman with a ruptured PcomA aneurysm. This aneurysm was treated by coil embolization with $20.6 \%$ of the final VER and recurrence at 6 months after treatment. a Pre-op intraoperative image. $\mathbf{b}$

Postoperative image. c Follow-up image. We created a virtual postcoiling model (VM) (d) and a real post-coiling model (RM) (g). The contour lines of the pressure difference of VM (e) and RM (h) are shown in the figures, and the high pressure difference $(\mathrm{PD})$ area is near the branch vessels. The PD values of this case were 3.05 in $\mathrm{VM}$ and 3.43 in RM. The streamlines of VM (f) and RM (i) are shown in the figures, and the white arrow indicates the coil plane. Blood flow collides with the coil plane and branches
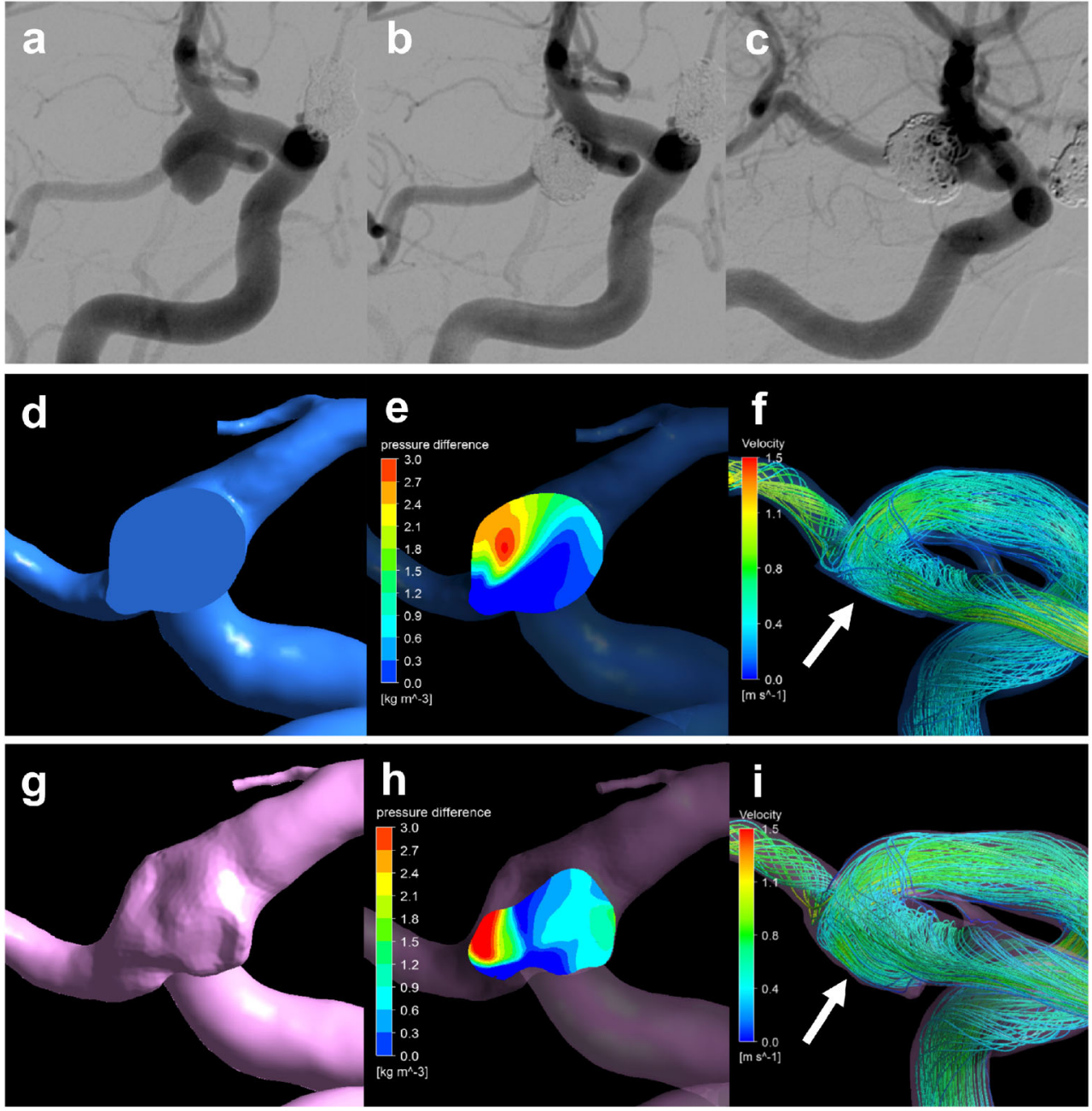

strategy. One method is to drastically change the surgical method from endovascular coil embolization to craniotomy clipping. Clipping is invasive but is known to have a low recurrence rate [3-5]. The recurrence rate would be reduced if patient consent was obtained and clipping treatment was selected. If coil embolization is selected even if the recurrence rate is expected to be high, stent-assisted techniques may be recommended. Previous reports have shown that stentassisted coil embolization decreases recanalization [23-25]. The underlying mechanism could be as follows: (1) reduced inflow to the aneurysm due to stenting, which promotes thrombus formation; (2) hemodynamic changes due to the straightening effect of the parent artery; and (3) promoting neointimal growth with stents. In cases where high PD as calculated using RM and a high recurrence rate are expected, more frequent follow-ups and additional treatments will be considered, which would lead to a change in treatment policy and would be useful information for the patients. Particularly, in cases of ruptured aneurysms, treatment is often performed as soon as possible after the onset, and it is difficult to calculate PD using VM before treatment. In our results, comparing the results of PD calculated using $\mathrm{RM}$ and VM, the results of RM show slightly stronger correlation with aneurysm recurrence than those of VM, and the use of RM is preferred for postoperative follow-up. Furthermore, RM reflects the actual postoperative coil plane, allowing for less biased and objective measurements.

Although clear geometry is essential for CFD analysis, obtaining sufficient quality of the geometry is technically difficult for aneurysms treated with coils. In this study, we used the TOF-MRA data of the actual coil plane after coil embolization to create RM. 3D-RA data are often used for CFD analysis because of the high resolution and accurate vessel visualization. However, TOF-MRA is more often used as a follow-up image than 3D-RA and contrast-enhanced magnetic resonance angiography (CE-MRA) because of its low invasiveness $[26,27]$. Ferre et al. reported on the utility of TOFMRA with DSA in 51 cases and concluded that TOF-MRA was at least as efficient as DSA for the evaluation of intracranial aneurysm occlusion [28]. In addition, van Amerongen et al. performed a systematic review and meta-analysis and reported that TOF-MRA can be used to study recurrence with 
Fig. 5 Illustrative stable unruptured paraclinoid aneurysm in a 44-year-old woman. The aneurysm was treated by coil embolization with $23.3 \%$ of the final VER. a Pre-op intraoperative image. b Postoperative image. c Follow-up image. We created a virtual post-coiling model (VM) (d) and a real post-coiling model (RM) (g). The contour lines of the pressure difference of VM (e) and RM (h) are depicted in the figures, and the high pressure difference (PD) area is proximal to the coil plane. The PD value in this case was 2.65 in VM and 2.80 in RM. The streamlines of VM (f) and RM (i) are depicted in the figures, and the white arrow indicates the coil plane. Blood flow along the coil plane
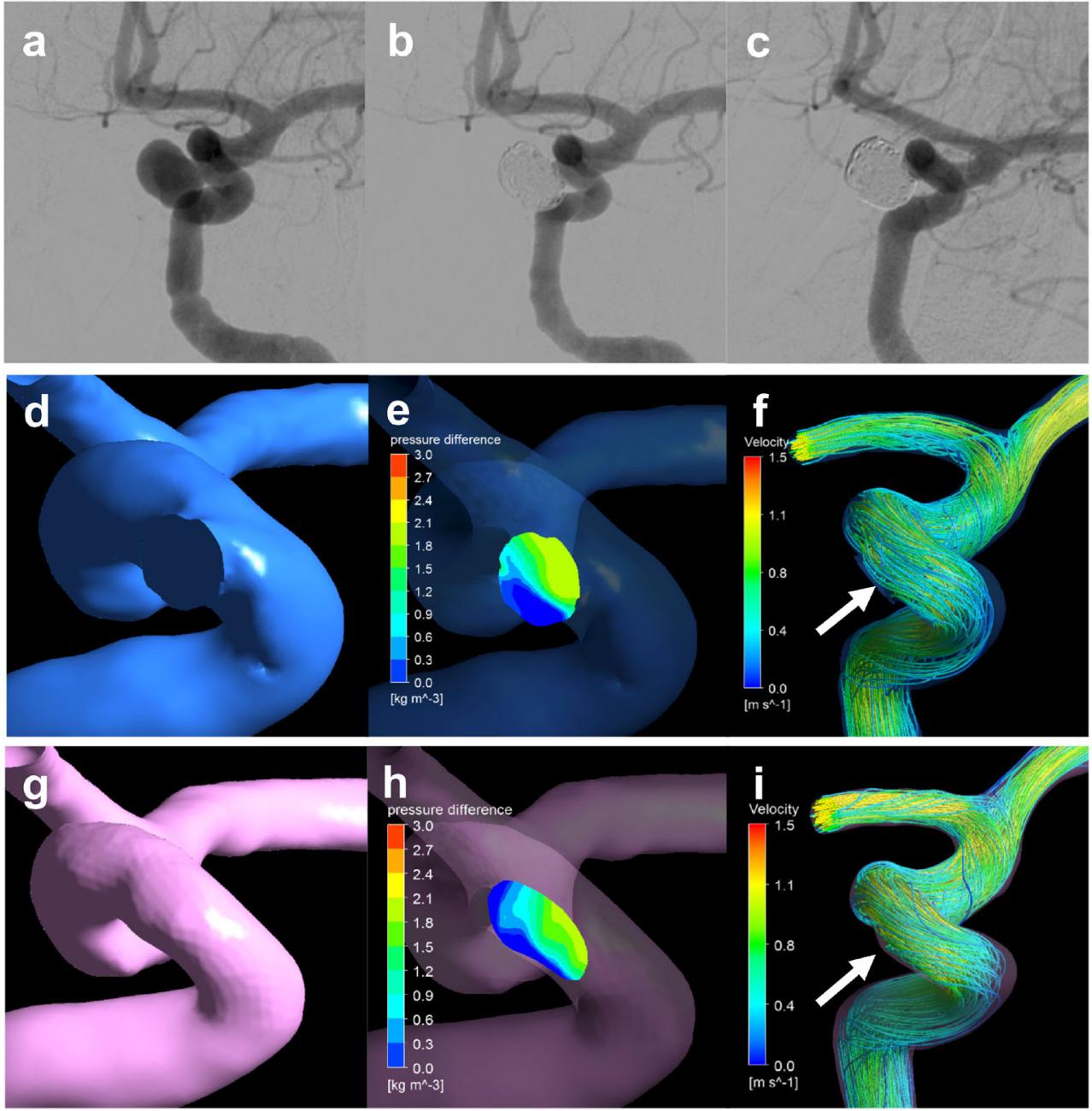

accuracy comparable with that of DSA. Moreover, they also reported that TOF-MRA is superior to CE-MRA for the follow-up of intracranial aneurysms treatment with endovascular coil occlusion [29]. It is beneficial to use TOFMRA during the follow-up of recurrence after coiling in clinical practice, and it is also a useful modality to evaluate the coil plane used in a model representing post-coil embolism. There have been several reports on CFD studies on the use of MRA images, and we believe that MRA data are useful for performing CFD analysis [30, 31].

\section{Limitations}

This study has several limitations. First, CFD was performed for only 50 aneurysms in this study. We should perform CFD analysis for many more cases to increase reliability. Second, we evaluated only aneurysms located in the ICA. We should consider those located in other parts such as middle cerebral artery and anterior communicating artery. Third, although it may vary from patient to patient, the boundary condition was uniform in all patients. To analyze the characteristics specific to the patients, there is a method in which boundary conditions are obtained and analyzed using phase-contrast magnetic resonance imaging and transcranial Doppler ultrasonic examination. Fourth, the RM construction was examined using the most recent MRA data after coil embolization, but the degree of thrombus formation in the aneurysm may differ depending on the duration until imaging. The effect of thrombosis may need to be taken into account when considering the coil plane that actually causes recurrence. Fifth, TOF-MRA has been used as the first-line modality in the current follow-up, and there has been no issues regarding quality; however, evaluation with a more accurate modality is required. To express a more accurate coil plane, it is necessary to consider data from modalities generating fewer artifacts, such as silent MRA [32]. Sixth, although PD in RM correlates with PD in VM, $R=0.698$ is not a strong correlation. PD in RM and PD in VM are hemodynamic parameters calculated from different modalities data and need to be thoroughly examined with respect 
to their correlation [33]. Further study is needed to reveal which is close to ground truth values of PD. Finally, this was a retrospective study; however, to confirm the relationship between PD and recurrence, prospective studies such as interventions in patients with high preoperative PD detection are required.

\section{Conclusions}

The CFD analysis data obtained using RM showed correlation with those obtained using VM. The analysis data in RM also showed that a high PD at the coil plane was strongly associated with recurrence, similar to that calculated using VM. PD can be considered clinically useful to predict recurrence after coil embolization without the need for invasion in patients.

Funding This research did not receive any specific grant from founding agencies in the public, commercial, or not-for-profit sectors.

\section{Compliance with ethical standards}

Conflict of interest disclosure The authors declare that they have no conflict of interest.

Ethical approval All procedures performed in studies involving human participants were in accordance with the ethical standards of the institutional and/or national research committee and with the 1964 Helsinki declaration and its later amendments or comparable ethical standards.

Informed consent Informed consent was obtained from all individual participants included in the study.

Open Access This article is licensed under a Creative Commons Attribution 4.0 International License, which permits use, sharing, adaptation, distribution and reproduction in any medium or format, as long as you give appropriate credit to the original author(s) and the source, provide a link to the Creative Commons licence, and indicate if changes were made. The images or other third party material in this article are included in the article's Creative Commons licence, unless indicated otherwise in a credit line to the material. If material is not included in the article's Creative Commons licence and your intended use is not permitted by statutory regulation or exceeds the permitted use, you will need to obtain permission directly from the copyright holder. To view a copy of this licence, visit http://creativecommons.org/licenses/by/4.0/.

\section{References}

1. Liu A, Huang J (2015) Treatment of intracranial aneurysms: clipping versus coiling. Curr Cardiol Rep 17(9):628

2. Molyneux AJ, Kerr RS, Birks J, Ramzi N, Yarnold J, Sneade M, Rischmiller J, Collaborators ISAT (2009) Risk of recurrent subarachnoid haemorrhage, death, or dependence and standardised mortality ratios after clipping or coiling of an intracranial aneurysm in the international Subarachnoid Aneurysm Trial (ISAT): longterm follow-up. Lancet Neurol 8(5):427-433
3. Investigators $C$ (2006) Rates of delayed rebleeding from intracranial aneurysms are low after surgical and endovascular treatment. Stroke 37(6):1437-1442

4. Raymond J, Guilbert F, Weill A, Georganos SA, Juravsky L, Lambert A, Lamoureux J, Chagnon M, Roy D (2003) Long-term angiographic recurrences after selective endovascular treatment of aneurysms with detachable coils. Stroke 34(6):1398-1403

5. Spetzler RF, McDougall CG, Albuquerque FC, Zabramski JM, Hills NK, Partovi S, Nakaji P, Wallace RC (2013) The barrow ruptured aneurysm trial: 3-year results. J Neurosurg 119(1):146157

6. Misaki K, Uchiyama N, Nambu I, Aida Y, Kamide T, Mohri M, Ueda F, Nakada M (2016) Optimizing the volume of the initial framing coil to facilitate tight packing of intracranial aneurysms. World Neurosurg 90:397-402

7. Sugiyama S, Niizuma K, Sato K, Rashad S, Kohama M, Endo H, Matsumoto Y, Ohta M, Tominaga T (2016) Blood flow into basilar tip aneurysms: a predictor for recanalization after coil embolization. Stroke 47(10):2541-2547

8. Hua Y, Oh JH, Kim YB (2015) Influence of parent artery segmentation and boundary conditions on hemodynamic characteristics of intracranial aneurysms. Yonsei Med J 56(5):1328-1337

9. Nambu I, Misaki K, Uchiyama N, Mohri M, Suzuki T, Takao H, Murayama Y, Futami K, Kawamura T, Inoguchi Y, Matsuzawa T, Nakada M (2019) High pressure in virtual postcoiling model is a predictor of internal carotid artery aneurysm recurrence after coiling. Neurosurgery 84(3):607-615

10. Takao H, Murayama Y, Otsuka S, Qian Y, Mohamed A, Masuda S, Yamamoto M, Abe T (2012) Hemodynamic differences between unruptured and ruptured intracranial aneurysms during observation. Stroke 43(5):1436-1439

11. Misaki K, Takao H, Suzuki T, Nishimura K, Kan I, Yuki I, Ishibashi T, Yamamoto M, Murayama Y (2017) Estimated pretreatment hemodynamic prognostic factors of aneurysm recurrence after endovascular embolization. Technol Health Care 25(5):843850

12. Kamide T, Misaki K, Nambu I, Mohri M, Uchiyama N, Nakada M (2017) Delayed asymptomatic coil migrations toward different arteries after aneurysmal embolization: case report. Acta Neurochir 159(3):593-598

13. Yoshiki K, Misaki K, Nambu I, Fukui I, Mohri M, Uchiyama N, Nakada M (2017) Intraoperative rupture of unruptured cerebral aneurysm during craniotomy: a case report. Case Rep Neurol 9(3):261-266

14. Steinman DA, Hoi Y, Fahy P, Morris L, Walsh MT, Aristokleous N, Anayiotos AS, Papaharilaou Y, Arzani A, Shadden SC, Berg P, Janiga G, Bols J, Segers P, Bressloff NW, Cibis M, Gijsen FH, Cito S, Pallarés J, Browne LD, Costelloe JA, Lynch AG, Degroote J, Vierendeels J, Fu W, Qiao A, Hodis S, Kallmes DF, Kalsi H, Long Q, Kheyfets VO, Finol EA, Kono K, Malek AM, Lauric A, Menon PG, Pekkan K, Esmaily Moghadam M, Marsden AL, Oshima M, Katagiri K, Peiffer V, Mohamied Y, Sherwin SJ, Schaller J, Goubergrits L, Usera G, Mendina M, Valen-Sendstad K, Habets DF, Xiang J, Meng H, Yu Y, Karniadakis GE, Shaffer N, Loth F (2013) Variability of computational fluid dynamics solutions for pressure and flow in a giant aneurysm: the ASME 2012 summer bioengineering conference CFD challenge. J Biomech Eng 135(2): 021016

15. Ford MD, Alperin N, Lee SH, Holdsworth DW, Steinman DA (2005) Characterization of volumetric flow rate waveforms in the normal internal carotid and vertebral arteries. Physiol Meas 26(4): 477-488

16. Campi A, Ramzi N, Molyneux AJ, Summers PE, Kerr RS, Sneade M, Yarnold JA, Rischmiller J, Byrne JV (2007) Retreatment of ruptured cerebral aneurysms in patients randomized by coiling or 
clipping in the International Subarachnoid Aneurysm Trial (ISAT). Stroke 38(5):1538-1544

17. Qian Y, Takao H, Umezu M, Murayama Y (2011) Risk analysis of unruptured aneurysms using computational fluid dynamics technology: preliminary results. AJNR Am J Neuroradiol 32(10):19481955

18. Cebral JR, Sheridan M, Putman CM (2010) Hemodynamics and bleb formation in intracranial aneurysms. AJNR Am J Neuroradiol 31(2):304-310

19. Russell JH, Kelson N, Barry M, Pearcy M, Fletcher DF, Winter CD (2013) Computational fluid dynamic analysis of intracranial aneurysmal bleb formation. Neurosurgery 73(6):1061-1068

20. Suzuki T, Stapleton CJ, Koch MJ, Tanaka K, Fujimura S, Suzuki T, Yanagisawa T, Yamamoto M, Fujii Y, Murayama Y, Patel AB (2019) Decreased wall shear stress at high-pressure areas predicts the rupture point in ruptured intracranial aneurysms. J Neurosurg 15:1-7

21. Suzuki T, Takao H, Suzuki T, Kambayashi Y, Watanabe M, Sakamoto H, Kan I, Nishimura K, Kaku S, Ishibashi T, Ikeuchi S, Yamamoto M, Fujii Y, Murayama Y (2016) Determining the presence of thin-walled regions at high-pressure areas in unruptured cerebral aneurysms by using computational fluid dynamics. Neurosurgery 79(4):589-595

22. Fujimura S, Takao H, Suzuki T, Dahmani C, Ishibashi T, Mamori H, Yamamoto M, Murayama Y (2018) A new combined parameter predicts re-treatment for coil-embolized aneurysms: a computational fluid dynamics multivariable analysis study. J Neurointerv Surg 10(8):791-796

23. Ishii A, Chihara H, Kikuchi T, Arai D, Ikeda H, Miyamoto S (2017) Contribution of the straightening effect of the parent artery to decreased recanalization in stent-assisted coiling of large aneurysms. J Neurosurg 127(5):1063-1069

24. Zenteno MA, Santos-Franco JA, Freitas-Modenesi JM, Gomez C, Murillo-Bonilla L, Aburto-Murrieta Y, Díaz-Romero R, Nathal E, Gómez-Llata S, Lee A (2008) Use of the sole stenting technique for the management of aneurysms in the posterior circulation in a prospective series of 20 patients. J Neurosurg 108(6):1104-1118

25. Feng MT, Wen WL, Feng ZZ, Fang YB, Liu JM, Huang QH (2016) Endovascular embolization of intracranial aneurysms: to use stent(s) or not? Systematic review and meta-analysis. World Neurosurg 93:271-278

26. Ahmed SU, Mocco J, Zhang X, Kelly M, Doshi A, Nael K, Doshi A, Nael K, De Leacy R (2019) MRA versus DSA for the follow-up imaging of intracranial aneurysms treated using endovascular techniques: a meta-analysis. J Neurointerv Surg 11(10):1009-1014

27. Bakker NA, Westerlaan HE, Metzemaekers JD, van Dijk JM, Eshghi OS, Mooij JJ, Groen RJ (2010) Feasibility of magnetic resonance angiography (MRA) follow-up as the primary imaging modality after coiling of intracranial aneurysms. Acta Radiol 51(2): 226-232

28. Ferre JC, Carsin-Nicol B, Morandi X, Carsin M, de Kersaint-Gilly A, Gauvrit JY, Desal HA (2009) Time-of-flight MR angiography at $3 \mathrm{~T}$ versus digital subtraction angiography in the imaging follow-up of 51 intracranial aneurysms treated with coils. Eur J Radiol 72(3): 365-369

29. van Amerongen MJ, Boogaarts HD, de Vries J, Verbeek AL, Meijer FJ, Prokop M, Bartels RH (2014) MRA versus DSA for follow-up of coiled intracranial aneurysms: a meta-analysis. AJNR Am J Neuroradiol 35(9):1655-1661

30. Sugiyama SI, Endo H, Omodaka S, Endo T, Niizuma K, Rashad S, Nakayama T, Funamoto K, Ohta M, Tominaga T (2016) Daughtersac formation related to blood inflow jet in an intracranial aneurysm. World Neurosurg 96:396-402

31. Kono K, Terada T (2014) Flow visualization of recurrent aneurysms after coil embolization by 3D phase-contrast MRI. Acta Neurochir 156(11):2035-2040

32. Ryu KH, Baek HJ, Moon JI, Choi BH, Park SE, Ha JY, Park H, Kim SS, Kim JS, Cho SB, Carl M (2020) Usefulness of noncontrast-enhanced silent magnetic resonance angiography (MRA) for treated intracranial aneurysm follow-up in comparison with time-of-flight MRA. Neurosurgery 87:220-228

33. Naito T, Miyachi S, Matsubara N, Isoda H, Izumi T, Haraguchi K, Takahashi I, Ishii K, Wakabayashi T (2012) Magnetic resonance fluid dynamics for intracranial aneurysms-comparison with computed fluid dynamics. Acta Neurochir 154(6):993-1001

Previous presentation 2018 CNS Annual Meeting, 6-10 October 2018, Houston, Texas.

Publisher's note Springer Nature remains neutral with regard to jurisdictional claims in published maps and institutional affiliations. 